\title{
Type-Spread Molecular Communications: Principles and Inter-Symbol Interference Mitigation
}

\author{
Weidong Gao, Terrence Mak, and Lie-Liang Yang \\ School of ECS, University of Southampton, SO17 1BJ, United Kingdom \\ Tel: 0044-(0)23-8059 3364, Email:wg1y15,tmak,lly@ecs.soton.ac.uk, http://www.ecs.soton.ac.uk
}

\begin{abstract}
Diffusion-based Molecular Communication (DMC) is a feasible method for information transmission in some nanonetworks operated in gas or liquid environments. In this paper, we first propose an information modulation scheme for DMC, which is referred to as the Type-Spread Molecular Shift Keying (TSMoSK). Considering that DMC signals usually experience severe inter-symbol interference (ISI), our TS-MoSK is characterized by introducing extra types of molecules for ISI mitigation (ISIM). Furthermore, we introduce two ISIM methods to the TS-MoSK modulated DMC systems, which are the active ISIM and passive ISIM. We detail their operation principles, and investigate as well as compare their achievable performance. Our studies show that, aided by the extra types of molecules, TS-MoSK outperforms the MoSK without spreading. Both ISIM approaches are effective for further improving the performance of TS-MoSK.
\end{abstract}

Index Terms-Diffusive molecular communication, molecular shift keying, spreading, inter-symbol interference, inter-symbol interference mitigation, interference cancellation.

\section{INTRODUCTION}

Diffusion-based Molecular Communication (DMC) is an essential information transmission approach between the nanomachines deployed in gas or in liquid medium environments [1, 2]. In these communications environments, at macro-scale, information molecules relying on the Brownian motion always migrate from a higher concentration region to a lower concentration region following Fick's law. Correspondingly, in literature, there are a range of contributions considered the channel modeling in DMC. For example, in [2,3], the noise modeling in DMC has been addressed. The studies show that noise in DMC cannot be considered separately from transmitted signal as done in the conventional radio-wave based communications systems, but is dependent on the transmitted signal. Furthermore, noise in DMC is non-stationary.

Based on the channel characteristics and noise models derived for DMC, three fundamental data modulation schemes have been developed in references for DMC, namely the Concentration Shift Keying (CSK), Pulse Position Modulation (PPM), and the Molecular Shift Keying (MoSK). First, CSK makes use of the concentration levels to convey information [4]. PPM restores received information based on the different time shifts of the concentration pulses appearing at receiver [5]. Finally, information in MoSK is encoded to a type of molecules via activating one out of several types of molecules [1]. In DMC, all these modulation schemes may experience intersymbol interference. However, MoSK has certain capability to mitigate ISI, owing to the employment of multiple types of molecules, and hence the probability of continuously activating the same type of molecules is less than one, which becomes smaller as the number of types increases. In this paper, on the basis of MoSK, we further enhance its ISI mitigation (ISIM) capability by introducing some extra types of molecules, and hence spreading the original MoSK signal. Therefore, the proposed scheme is referred to as the Type-Spread MoSK (TSMoSK). Following the principles of MoSK, our studies show that TS-MoSK is able to improve the ISIM capability of MoSK, and hence enhance its error performance, especially in the case that there is severe ISI. The cost for this performance improvement is the increased types of molecules, which may be exploit for other purpose, such as, to simultaneously support multiple communication links.

A main challenge in DMC is that the slow molecular diffusion process results in significant propagation delay spread, generating serious ISI. Therefore, a lot of effort has been made to design the detection techniques for DMC systems, which are robust to ISI or have the capability to mitigate ISI. As some examples, the traditional signal processing methods, such as Maximum A-Posteriori (MAP), Maximum Likelihood (ML), etc., have been introduced to equalize the DMC channels [6,7], in order to achieve optimum performance. In order to reduce the implementational complexity of the optimum equalizers, adaptive Decision-Feedback Equalizer (DFE) and Minimum MeanSquare Error (MMSE) equalizer have been considered [8,9]. In terms of ISIM and detection reliability, these detection (equalization) algorithms are efficient. However, it is highly challenging to implement them at nano-scale. Considering the research background and the feasibility of implementation in practice, in this paper, we consider only low low-complexity detection and introduce two ISIM methods, namely, the passive ISIM and active ISIM. As shown in our forthcoming discourses, the passive ISIM is implemented at receiver, which is the extension of the ISIM approach proposed in [7] and improved in $[10,11]$. By contrast, the proposed active ISIM is operated at transmitter, which controls the number of molecules emitted for transmitting a symbol with reference to the expected ISI experienced by the symbol. Our studies show that the TSMoSK enhanced by the active ISIM is efficient in short-distance communication scenarios, but is invalid if the communication distance is too long. By contrast, the passive ISIM has the capability to boost the error performance of the TS-MoSK assisted DMC systems in any communication scenarios addressed in 
this paper.

The remainder of the paper is organized as follows. Section II introduces the DMC system model and the principles of TSMoSK. In Section III, we present principles of the two ISIM techniques. The performance results and their analysis are addressed in Section IV. Finally, the main conclusions from research are summarized in Section V.

\section{System Model of Type-Spread Molecular COMMUNICATION}

The idea behind TS-MoSK is that $Q(Q>M)$ types of molecules are used to transmit $\log _{2} M$ bits per symbol using MoSK modulation. Hence, information is spread on the types of molecules, and the use of extra types of molecules makes the probability that the same type of molecules is continuously emitted becomes smaller, which naturally mitigates the effect of ISI. Below we describe the principles of TS-MoSK.

\section{A. Transmitter Scheme}

Let us assume that there is a binary data sequence to be transmitted. At the transmitter, this binary data sequence can be divided into symbols, forming a symbol sequence $x^{T}=$ $\left[x_{0}, x_{1}, \ldots, x_{j}, \ldots x_{N}\right]$, where $N$ is the number of symbols, $x_{i} \in\{0,1, \ldots, M-1\}$ is an integer, representing $\log _{2} M$ bits of the $i$ th symbol in the binary data sequence. Then, when the $M$-ary MoSK modulation [12] is employed, these $N$ symbols are transmitted one-by-one in serial based on the principles of MoSK. Specifically, for transmitting $x_{i}$, a pulse of $x_{i}$-th type of molecules is emitted by the molecular transmitter.

When the TS-MoSK is considered, we assume that there are $Q(Q>M)$ types of molecules are used to transmit the $M$ ary symbols. In order to configure the $M$-ary symbols to the $Q$ types of molecules, let us introduce a periodic spreading sequence, expressed as $\boldsymbol{q}^{T}=\left[q_{0}, q_{1}, \ldots, q_{i}, \ldots, q_{N}\right]$, where $q_{i} \in\{0,1, \ldots, Q-1\}$. With the aid of this spreading sequence, then, the $M$-ary symbols can be mapped to the $Q$-ary symbols by a simple operation, such as,

$$
\begin{aligned}
\boldsymbol{y} & =\left[y_{0}, y_{1}, \ldots, y_{i}, \ldots, y_{N}\right]^{T} \\
& =(\boldsymbol{x}+\boldsymbol{q}) \bmod Q
\end{aligned}
$$

where $\bmod Q$ means modulo- $Q$ operation. Hence, $y_{i}$ is an integer taking a value in $[0, Q-1]$. Finally, the elements of $\boldsymbol{y}$ are transmitted one-by-one in the principles of a $Q$-ary MoSK. In detail, for the $i$ th element of $\boldsymbol{y}$, a pulse of the $y_{i}$-th type of molecules is emitted at the beginning of a symbol interval, while all the other types of molecules maintain inactive.

\section{B. Channel Model}

We assume that information molecules released by the transmitter are propagated over a DMC channel. As shown in [13], given a spherical receiver having a fixed distance $r$ from the transmitter, the concentration observed in the receiver meets the Fick's law, having the time-dependent function of

$$
c(t)=\frac{A}{(4 \pi D t)^{\frac{3}{2}}} \exp \left(-\frac{r^{2}}{4 D t}\right), t \geq 0
$$

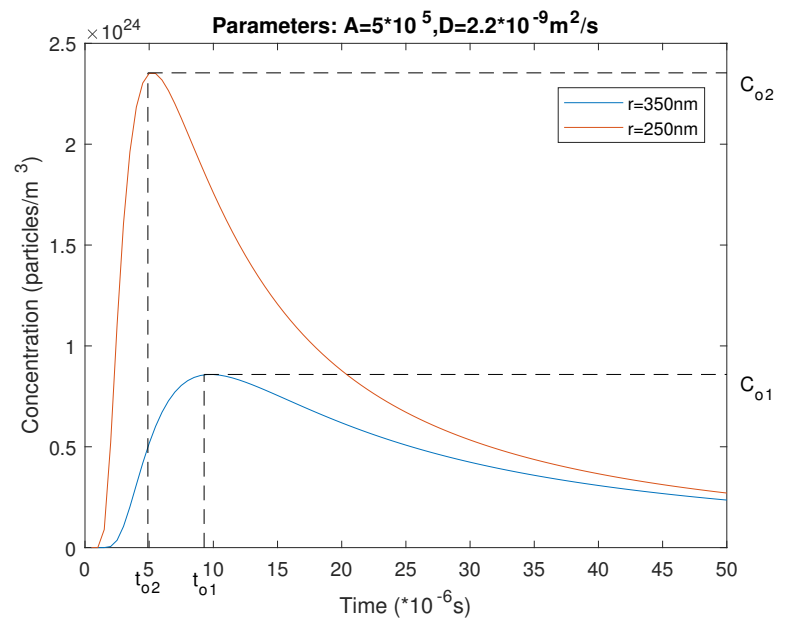

Fig. 1. Function of molecular concentration.

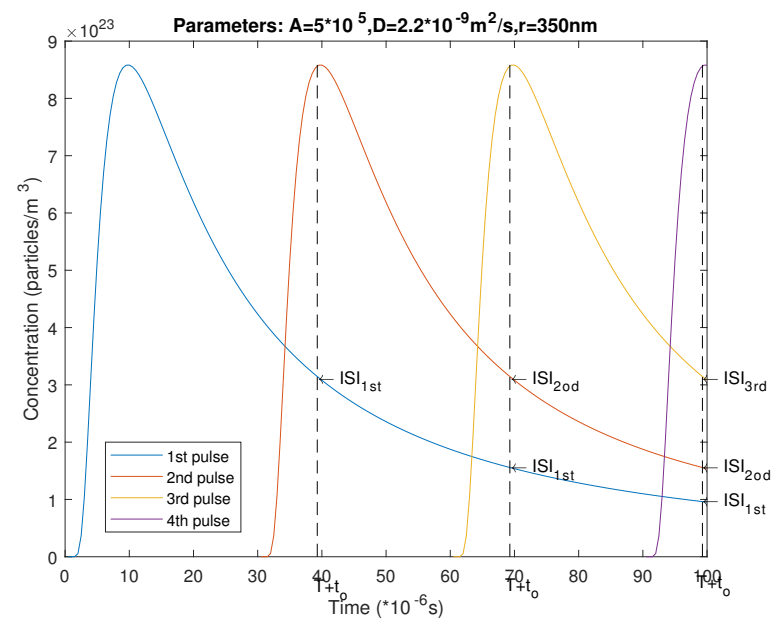

Fig. 2. Demonstration of inter-symbol interference.

when a pulse of $A$ molecules is emitted at $t=0$. In (2), $D$ is the diffusion coefficient of communication medium, and $t$ is the propagation time.

Fig. 1 demonstrates the concentration function in one symbol interval for given values of $D, A$ and $r$, as shown in the figure. In this concentration function, the peak point can be found from the extremum point of (2) by solving the equation of $d c(t) / d t=$ 0 , which gives $t_{o}=r^{2} / 6 D$. Substituting this point into (2), we obtain the maximum value of concentration of

$$
c_{o}=\left(\frac{3}{2 \pi e}\right)^{\frac{3}{2}} \frac{A}{r^{3}}
$$

As shown in Fig. 1, the received pulse is not constrained within one symbol duration, but extend, in theory, to infinity. Hence, when symbols are continuously transmitted, as shown in Fig. 2, the pulses corresponding to different symbols overlap with each other, generating ISI. Explicitly, the strength of ISI is dependent of the symbol rate, or on the symbol duration, higher 
symbol rate has higher ISI. However, from Figs. 1 and 2, we can be implied that the ISI on a specific symbol is dominated by the several pulses (or symbols) transmitted right in the front of the symbol. Hence, in order to mitigate ISI, we should try to avoid transmitting the same type of molecules continuously. MoSK modulation has this embedded merit, and a higher modulation order, i.e., the value of $M$, yields a lower probability of transmitting the same type of molecules continuously. In this paper, we enhance this by introducing some extra types of molecules for information transmission, which can further reduce the above-mentioned probability, and hence the ISI.

\section{Received Signal}

In order to detect the data, the observation about the molecular concentration at the receiver can be utilized. Specifically, for detecting the $k$ th symbol, the decision variables can be obtained by sampling the concentrations of the $Q$ types of molecules at $t=k T+t_{o}$, which can be expressed as

$$
\begin{aligned}
r_{q}(k)= & r_{q}\left(t=k T+t_{o}\right) \\
= & \sum_{i=0}^{\min \{I, k\}} l_{q, k-i} c_{q}\left(i T+t_{o}\right)+n_{q}\left(k T+t_{o}\right) \\
& =l_{q, k} c_{q, o}+\underbrace{\sum_{i=1}^{\min \{I, k\}} l_{q, k-i} c_{q, i}}_{\text {ISI }}+n_{q, k}, \\
& q=0,1, \ldots, Q-1 ; k=0,1, \ldots, N
\end{aligned}
$$

for the $Q$ types of molecules. In (4), the ISI length is assumed to be $I, l_{q, i}$ is a logic value, which is ' 1 ', if the $q$ th type of molecules is activated during the $i$ th symbol duration, and is ' 0 ', if the $q$ th type of molecules is inactive during the $i$ th symbol duration. In (4), $c_{q, i}$ is the expected concentration of the $q$ th type of molecules at $t=i T+t_{o}$, when a pulse of $A$ molecules was sent at $t=0$. Finally, $n_{q}(k)$ is the noise resulted from Brownian motion of the $q$ type of molecules, which can be approximately treated as the Gaussian noise, obeying the distribution with mean zero and a variance of [13]

$$
n_{q, k} \sim \mathcal{N}\left(0, \frac{1}{V} \sum_{i=0}^{\min \{I, k\}} l_{q, k-i} c_{q, i}\right)
$$

where $V=\frac{4}{3} \pi \rho^{3}$ is the volume of a spherical receiver with a radius $\rho$.

Based on the decision variables shown in (4), the detection of the $k$ th symbol can be carried out as follows. First, by comparing the $Q$ decision variables as shown in (4), the type of molecules activated for transmitting the $k$ th symbol can be identified as the one having the highest concentration, which can be formulated as

$$
S_{k}=\arg \max _{q \in\{0,1, \ldots, Q-1\}}\left\{r_{q}(k)\right\}, k=0,1, \ldots, N
$$

Then, corresponding to the modulo addition of (1) carried out at the transmitter, an estimate to the $M$-ary symbol $x_{k}$ can be obtained by a modulo subtraction operation on $S_{k}$, which can be expressed as

$$
\hat{x}_{k}=\left(S_{k}-q_{k}\right) \quad \bmod Q, k=0,1, \ldots, N
$$

Finally, upon mapping $\left\{\hat{x}_{0}, x_{1}, \ldots, x_{N}\right\}$ to binary, we obtain the estimates to the transmitted binary sequences.

From the operation of (7), we can know that the recovered $\hat{x_{k}}$ may not be in the range of $[0, M-1]$. In this case, there is an error occurred, and the receiver may either throw away the detected symbol, or randomly take a value in $[0, M-1]$ as the estimate to the received symbol.

\section{Inter-Symbol Interference Mitigation}

From our discussion in Section II-B, we can know that the proposed TS-MoSK may also experience ISI, which becomes more serious, when transmission rate increases. For instant, if the same type of molecules, such as Type $i$, is continuously transmitted several times, before the transmission of another type, say Type $j$, the accumulated concentration of Type $i$ molecules may be higher than that of Type $j$ molecules, hence resulting in error detection. Therefore, ISI mitigation in TSMoSK is also important. In this section, we introduce two approaches for ISI mitigation (ISIM), namely, the passive ISIM approach operated at receiver, and the active ISIM approach executed at transmitter.

\section{A. Passive ISI Mitigation}

A simple passive ISIM technique has been proposed in [7] for the DMC systems with On-Off Keying (OOK) modulation. This ISIM scheme can be directly extended to our TS-MoSK. In detail, instead of making the decision solely relying on the observations obtained at $t=k T+t_{o}$, which yields the decision variables as shown in (4), when the passive ISIM is employed, the receiver makes the decision depending on the observations at both $t=k T+t_{o}$ and $t=k T$, giving the decision variables to be

$$
\begin{aligned}
Z_{q}(k)= & r_{q}\left(t=k T+t_{o}\right)-r_{q}(t=k T) \\
= & \sum_{i=0}^{\min \{I, k\}} l_{q, k-i} c_{q}\left(i T+t_{o}\right)+n_{q}\left(k T+t_{o}\right) \\
& -\left(\sum_{i=1}^{\min \{I, k\}} l_{q, k-i} c_{q}(i T)+n_{q}(k T)\right) \\
& q=0,1, \ldots, Q-1 ; k=0,1, \ldots, N
\end{aligned}
$$

where $r\left(t=k T+t_{o}\right)$ is as the same as (4), while $r(t=k T)$ is the concentration observed at $t=k T$.

As shown in Fig. 1, $t=k T+t_{o}$ is time instant generating the peak molecular concentration, if a pulse of molecules is sent for the $k$ th symbol. By contrast, $r(t=k T)$ is the molecular concentration presenting at the beginning of the $k$ th symbol duration, at which point the pulse of molecules for the $k$ th symbol has not been emitted yet. Therefore, $r(t=k T)$ includes only the ISI from the molecules sent before $t=k T$, which can be used to approximate the ISI suffered by the $k$ th symbol at 
the sampling time $t=k T+t_{o}$. Consequently, after the ISIM as shown in (8), the detection can be expected to me more reliable.

From Figs. 1 and 2, we can be implied that the value of $r(t=k T)$ obtained at $t=k T$ may be slightly higher than the actual ISI experienced by the $k$ th symbol at $t=k T+t_{o}$, which results in excessive cancellation. This phenomenon becomes more declared, when the transmission rate becomes higher, making received pulses close to each other. Furthermore, as shown in (8), the ISIM operation introduces extra noise and hence yields noise amplification. In [11], an improved ISIM scheme has been proposed, which mitigates these side effects via downscaling the ISI estimated at $t=k T$.

\section{B. Active ISI Mitigation}

The passive ISIM is operated at receiver without any impact on the signal transmission and propagation. By contrast, active ISIM approaches are operated at the transmitter side via the corresponding adjustment of transmitted signals. In this section, we propose a novel active ISIM technique for the TS-MoSK, which can also be applied to many other DMC systems with various data modulation schemes.

In all our previous analysis and discussion, the number of molecules per pulse emitted by transmitter is a constant $A$, as seen in (2), which determines that the expected peak concentration $c_{o}$, if only single molecular pulse is emitted. When there is ISI, this peak value will change depending on the actual ISI. Since transmitter has the information to be transmitted, and hence knows exactly which and when the pulses are transmitted, the transmitter is capable of modifying the corresponding number of molecules to be emitted, so that the expected peak concentration is always $c_{o}$. Our active ISIM technique is based on this observation. Instead of always emitting $A$ molecules, the numbers of molecules actually emitted by the transmitter for sending the $k$ th symbol is adjusted to

$$
\begin{gathered}
A_{q, k}=\left\{\begin{array}{l}
A, \quad \text { when } k=0 \\
A-\frac{\sum_{i=1}^{\min \{I, k\}} l_{q, k-i} A_{q, k-i} c_{q, i}}{c_{q, o}},
\end{array}\right. \\
k=0,1, \ldots, N ; q=0,1, \ldots, Q-1
\end{gathered}
$$

where $c_{q, 0}, c_{q, 1}, \ldots, c_{q, I}$ are the concentration obtained from (2), when the number of molecules with a pulse if $A$.

It can be shown that, when the transmitter emits the pulses of molecules with the numbers of molecules of the pulses as shown in (9), the expected concentration of the type of molecules activated during the $k$ th symbol duration is always $c_{o}$ at the sampling instant of $t=k T+t_{o}$. This can be demonstrated as follows by assuming $k>I$. Let us assume that the $q$ th type of molecules is activated during the $k$ th symbol duration. Let the numbers of the $q$ th type of molecules sent corresponding to the symbols $x_{k-I}, x_{k-I+1}, \ldots, x_{k}$ be expressed as $l_{q, k-I} A_{q, k-I}, l_{q, k-I+1} A_{q, k-I+1}, \ldots, A_{q, k}$, where $l_{q, k}=1$ as the $q$ th type is assumed to be activated during the $k$ th symbol duration. Then, at the sampling time of $t=k T+t_{o}$, the expected concentration is

$$
\bar{c}_{q, k}=\frac{A_{q, k} c_{q, o}}{A}+\sum_{i=1}^{I} l_{q, k-i} \frac{A_{q, k-i} c_{q, i}}{A}
$$

Upon substituting $A_{q, k}$ with $k>I$ from (9) into the above equation, we can readily show that

$$
\bar{c}_{q, k}=c_{q, o}
$$

In comparison with the passive ISIM scheme introduced in Section III-A, we realize that the active ISIM technique has the following characteristics. First, the ISIM operation is carried out at transmitter, which shifts the complexity from receiver to transmitter. This is practically useful in the communications scenarios, where receivers are more capable than transmitters. Second, by making use of the expected ISI, the total number of molecules emitted is reduced, which in return, can be used to increase the signal-to-noise ratio (SNR), and ultimately improve reliability. Additionally, the reduced number of molecules also reduces the counting noise associated with both the current and following detection.

Below we provide some simulation results to characterize the performance of the TS-MoSK, as well as that of the TS-MoSK with respectively the passive and positive ISIM schemes.

\section{Performance Results}

In this paper, the error performance of the TS-MoSK assisted DMC systems is depicted against the SNR per bit. This SNR is defined as the ratio between the power received from a single pulse of molecules emitted for transmitting one bit and the noise power, formulated by $[11,14]$

$$
\mathrm{SNR}=\frac{c_{o}^{2}}{c_{o} / V}=V c_{o}
$$

In our simulations, for a given SNR per bit and the volume $V$ of a receiver, we can obtain $c_{o}$ from (12). Then, when the communications distance $r$ is given, from (3), we can derive the corresponding number of molecules $A_{b}$ for transmitting one bit. As $M$-ary MoSK is used in the TS-MoSK, the total number of molecules emitted for transmitting a symbol is $A=\log _{2} M \times A_{b}$.

In our simulations, some parameters are fixed, which are $D=2.2 \times 10^{-9} \mathrm{~m}^{2} / \mathrm{s}$ and $V=\frac{4}{3} \pi \rho^{3}$ with $\rho=1 \mathrm{~nm}$. The length of ISI is obtained via the measurement of

$$
I \triangleq \arg _{i}\left\{\frac{c_{i}}{c_{o}} \leq 0.01 \%\right\}
$$

meaning that only the ISI with the strength above $0.01 \%$ of the present desired signal is considered, the ISI weaker than this value is ignored. This is reasonable, as the ignored ISI is at least $80 \mathrm{~dB}$ lower than the desired signal.

First, in Fig. 3 we compare the BER performance of the DMC systems with the TS-MoSK operated with different levels of spreading, when a fixed 4-ary MoSK, and hence the bit rate of 2 bits/symbol, is considered. Furthermore, in the figure three symbol durations of $T$ are considered, which correspond to 


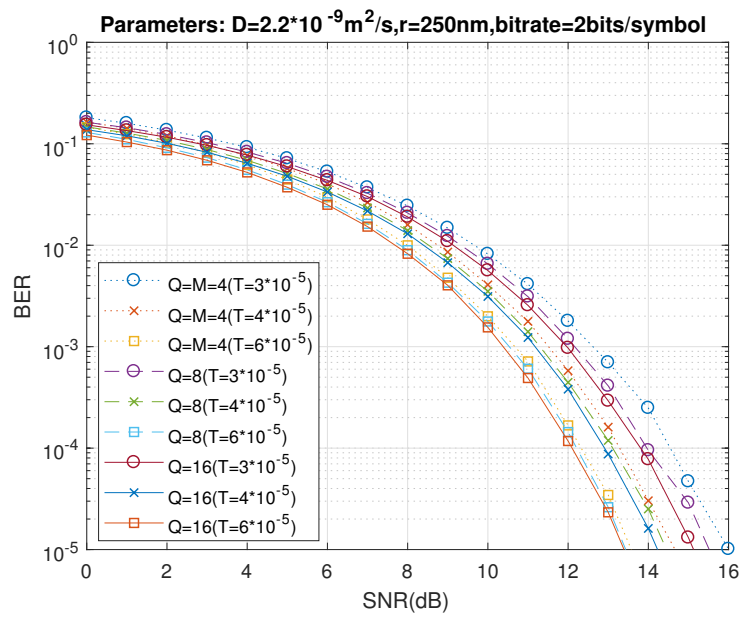

Fig. 3. BER versus SNR per bit performance of the DMC systems with 4-ary MoSK modulation and different TS levels at the same bit rate of 2 bits/symbol.

three symbol rates given by $R=1 / T$. From Fig. 3 we can have the following observations. First, the BER performance of the DMC systems improves, as the number of the types of molecules used for TS increases. Specifically, in the case of $T=3 \times 10^{-5} \mathrm{~s}$, explicit performance improvement can be observed, when the number of types is increased from $Q=4$ to $Q=8$, and then to $Q=16$. By contrast, when $T=6 \times 10^{-5} \mathrm{~s}$, only slight performance improvement is observed at relatively high SNR. Furthermore, we can be implied from the results that the performance improvement becomes less, as the symbol duration $T$ increase. The reason behind the above observation is that: a) MoSK modulation itself has certain capability to mitigate ISI, as previously mentioned, and b) as $T$ increases, ISI becomes shorter, making the effect of TS less observable. Second, in the case of $Q=M=4$, the BER performance improves, as the symbol duration increases, due to the reduced transmission rate. From this we can also be implied that, for a given value of $Q$, the BER performance improves, as the symbol duration increases.

Fig. 4 depicts the BER versus SNR performance of the TSMoSK assisted DMC systems with $Q=16$ types of molecules to transmit 4-ary MoSK symbols. Both the DMC systems without ISIM and that with passive ISIM are considered. Similar to the observations in Fig. 3, when the data rate is relatively high, yielding relatively severe ISI, employing passive ISIM is capable of generating noticeable performance gain. By contrast, when data rate becomes lower, ISI also becomes weak. Straightforwardly, the performance improvement resulted from passive ISIM becomes less significant.

In contrast to Fig. 4, in Fig. 5 we show the BER versus SNR performance of the TS-MoSK assisted DMC systems with $Q=16$ types of molecules to transmit 4-ary MoSK symbols, when without employing ISIM or with active ISIM. Note that, when the active ISIM is implemented, the number of molecules released per symbol is time variant, which is depended on the

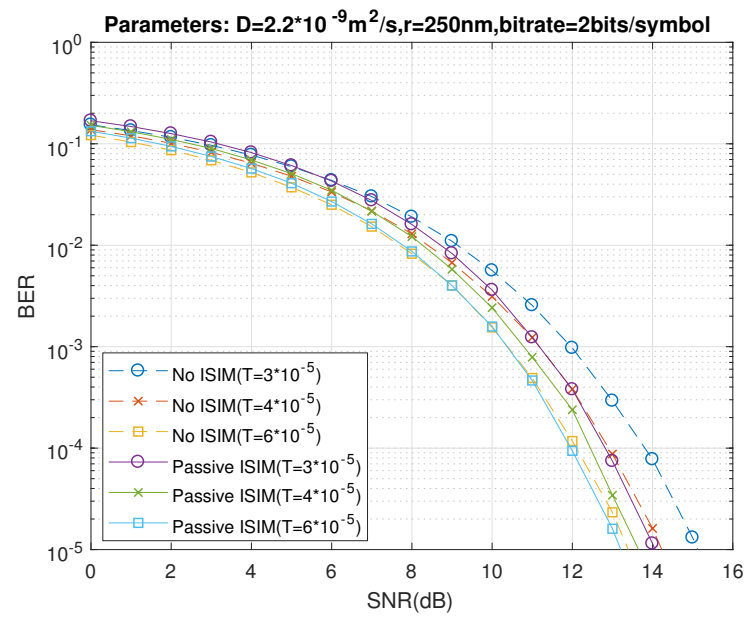

Fig. 4. BER versus SNR performance of DMC systems with the TS-MoSK supported by $Q=16$ types of molecules, when $4-$ ary MoSK is employed with/without passive ISIM.

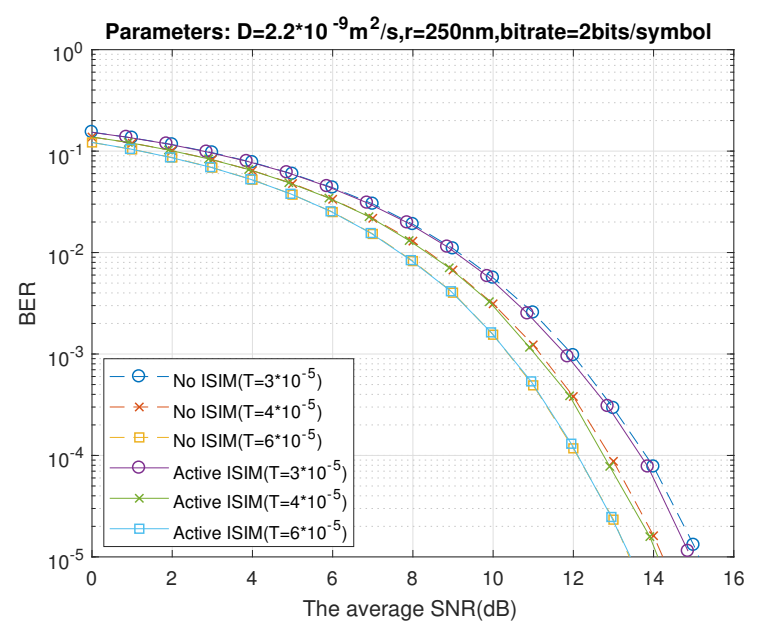

Fig. 5. BER versus SNR performance of DMC systems with the TS-MoSK supported by $Q=16$ types of molecules, when 4 - ary MoSK is employed with/without passive ISIM.

ISI. Hence, the SNR shown in the figure is the average SNR per bit, computed based on the actual number of molecules emitted by the transmitter. Specifically, in our simulations for the case of active ISIM, we set the number of molecules released during the first symbol period to be the same as that for the cases without ISIM or with passive ISIM. Therefore, the average SNR should be slightly lower than the SNR of the first symbol. The same as Fig. 4 for using passive ISISM, as demonstrated in Fig. 5, the active ISIM is capable of attaining some performance gain, when data rate is high, meaning that there is strong ISI. By contrast, when data rate is low, yielding smaller ISI, as expected, there performance gain also becomes smaller, and finally, there is no performance gain, when data rate is very low, making ISI ignorable.

Finally, in Fig. 6, we compare the BER versus transmission 


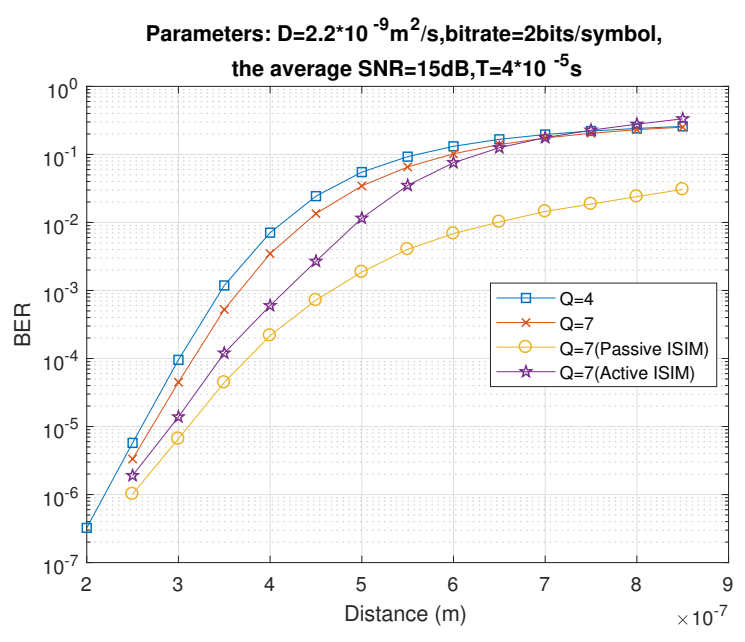

Fig. 6. Performance comparison of the 4-ary MoSK modulated DMC systems with various signaling and detection schemes considered.

distance performance of the DMC systems employing the various transmission and ISIM schemes, when the SNR per bit is fixed to $15 \mathrm{~dB}$ and the symbol interval to $T=4 \times 10^{-5} \mathrm{~s}$, while adjusting the transmission distance $r$ from $200 \mathrm{~nm}$ to $900 \mathrm{~nm}$. From the results of Fig. 6 we may have the following observations. First, as the propagation distance increases, the error performance of the DMC systems in any case degrades, due to the increased ISI. Second, at low to moderate transmission distance, we can observe that the TS-MoSK, TSMoSK with passive ISIM and the TS-MoSK with active ISIM all outperform the pure MoSK. Third, when the transmission distance becomes sufficiently long, the performance of the TS-MoSK converges to that of the original MoSK, implying that the benefit from TS disappear. This is because the severe ISI introduced by long transmission distance overwhelms the benefit obtained from TS. Fourth, at high transmission distance, such as $r>700 \mathrm{~nm}$, the active ISIM is even outperformed by the original MoSK, meaning that ISIM is useless. This is true because the received concentration pulse, as shown in Fig. 1, becomes flatter, as transmission distance increases. In this case, the reduced number of emitted molecules makes the detection hard. Finally, as shown in Fig. 6, at any transmission distance, the passive ISIM method is efficient, which significantly outperforms the active ISIM, if transmission distance is relatively long, and has a big performance gain over TS-MoSK for any transmission distance.

\section{CONCLUSIONS}

In this paper, we have first proposed a TS-MoSK scheme for improving the error performance of the MoSK by spreading the transmitted information over more types of molecules, and hence reducing the ISI. In order to further mitigate the ISI, we have introduced a passive ISIM operated at receiver, and proposed an active ISIM operated at transmitter. Both their advantages and disadvantages have been discussed. The performance of the MoSK modulated DMC systems employing respectively these schemes has been investigated. Our studies demonstrate that, with proper design and system settings, the capability of ISIM can be improved by the TS operation, which can be further, usually significantly, enhanced by either the active ISIM or the passive ISIM. When comparing active ISIM with passive ISIM, we find that the passive ISIM is usually more efficient than the active ISIM in terms of error performance. Active ISIM is only effective in short-distance communication scenario. However, active ISIM has the advantage that its impact on the communication environment is less than passive ISIM, owing to the reduced number of molecules emitted by the active ISIM into the communication environment. Our future research will consider the practical implementation of TSMoSK, and analyze the performance of the TS-MoSK assisted DMC systems with active/passive ISIM.

\section{REFERENCES}

[1] M. S. Kuran, H. B. Yilmaz, T. Tugcu, and I. F. Akyildiz, "Modulation techniques for communication via diffusion in nanonetworks," in 2011 IEEE International Conference on Communications (ICC), June 2011, pp. $1-5$.

[2] N. Garralda, I. Llatser, A. Cabellos-Aparicio, and M. Pierobon, "Simulation-based evaluation of the diffusion-based physical channel in molecular nanonetworks," in 2011 IEEE Conference on Computer Communications Workshops (INFOCOM WKSHPS), April 2011, pp. 443448.

[3] M. Pierobon, I. F. Akyildiz et al., "Diffusion-based noise analysis for molecular communication in nanonetworks," IEEE Transactions on Signal Processing, vol. 59, no. 6, pp. 2532-2547, 2011.

[4] B. Atakan and O. B. Akan, "Deterministic capacity of information flow in molecular nanonetworks," Nano Communication Networks, vol. 1, no. 1, pp. 31-42, 2010.

[5] A. Zare, A. Jamshidi, and A. Keshavarz-Haddad, "Receiver design for pulse position modulation technique in diffusion-based molecular communication," in 2017 IEEE 4th International Conference on KnowledgeBased Engineering and Innovation (KBEI), Dec 2017, pp. 0729-0733.

[6] C. T. Chou, "Maximum a-posteriori decoding for diffusion-based molecular communication using analog filters," IEEE Transactions on Nanotechnology, vol. 14, no. 6, pp. 1054-1067, Nov 2015.

[7] L. Meng, P. Yeh, K. Chen, and I. F. Akyildiz, "On receiver design for diffusion-based molecular communication," IEEE Transactions on Signal Processing, vol. 62, no. 22, pp. 6032-6044, Nov 2014.

[8] D. Kilinc and O. B. Akan, "Receiver design for molecular communication," IEEE Journal on Selected Areas in Communications, vol. 31, no. 12, pp. 705-714, December 2013.

[9] B. Tepekule, A. E. Pusane, H. B. Yilmaz, C. Chae, and T. Tugcu, "ISI mitigation techniques in molecular communication," IEEE Transactions on Molecular, Biological and Multi-Scale Communications, vol. 1, no. 2, pp. 202-216, June 2015.

[10] L. Shi and L. L. Yang, "Diffusion-based molecular communications: Inter-symbol interference cancellation and system performance," in 2016 IEEE/CIC International Conference on Communications in China (ICCC), July 2016, pp. 1-6.

[11] L. Shi and L. Yang, "Error performance analysis of diffusive molecular communication systems with on-off keying modulation," IEEE Transactions on Molecular, Biological and Multi-Scale Communications, pp. 1-1, 2018.

[12] A. W. Eckford, "Achievable information rates for molecular communication with distinct molecules," in 2007 2nd Bio-Inspired Models of Network, Information and Computing Systems, Dec 2007, pp. 313-315.

[13] A. Noel, K. C. Cheung, and R. Schober, "Improving receiver performance of diffusive molecular communication with enzymes," IEEE Transactions on NanoBioscience, vol. 13, no. 1, pp. 31-43, March 2014.

[14] D. Arifler, "Connectivity properties of free diffusion-based molecular nanoscale communication networks," IEEE Transactions on Communications, vol. 65, no. 4, pp. 1686-1695, April 2017. 